\title{
Embedding a Forest in a Graph
}

\author{
Mark K. Goldberg and Malik Magdon-Ismail \\ Department of Computer Science, \\ Rensselaer Polytechnic Institute \\ Troy, NY, 12180. \\ goldberg@cs.rpi.edu; magdon@cs.rpi.edu
}

Submitted: May 7, 2010; Accepted: Apr 23, 2011; Published: Apr 29, 2011

Mathematics Subject Classification: 05C35, 05C60

\begin{abstract}
For $p \geq 1$, we prove that every forest with $p$ trees whose sizes are $a_{1}, \ldots, a_{p}$ can be embedded in any graph containing at least $\sum_{i=1}^{p}\left(a_{i}+1\right)$ vertices and having minimum degree at least $\sum_{i=1}^{p} a_{i}$.
\end{abstract}

\section{Introduction}

It is a folklore fact that every tree with $d \geq 0$ edges can be embedded in any graph with minimum vertex degree $d$. Indeed, a linear algorithm to find such an embedding would sequentially embed the vertices of the tree according to a depth first search ordering of the tree vertices. It is likely, though, that the required bound on the minimum degree is excessive, as captured by the famous conjecture by Erdôs and Sós ([3]), which states that every tree with $d$ edges can be embedded in any graph whose average degree is greater than $d-1$. A number of results $([1,2,6,7,8,9])$ confirm the conjecture for some classes of trees and classes of graphs. The full conjecture is still neither proved, nor disproved.

A natural extension of the problem is to embed a forest in a graph. If $F=T_{1} \cup \cdots \cup T_{p}$ is a forest of $p$ disjoint trees whose sizes are $a_{1}, \ldots, a_{p}$ respectively, then a necessary condition for embedding $F$ in a graph $G$ is that $|V(G)| \geq \sum_{i=1}^{p}\left(1+a_{i}\right)$. The straightforward tree embedding algorithm outlined above may fail, even if the minimum degree is at least $\sum_{i=1}^{p} a_{i}$. However, we show that this condition on the minimum degree (in addition to the obvious necessary condition) is sufficient to guarantee that the forest can be embedded in the graph; we prove the following:

Theorem 1 Let $F=T_{1} \cup \cdots \cup T_{p}$ be a forest and $d=\sum_{i=1}^{p} a_{i}$, where $a_{i}$ is the number of edges in the tree $T_{i}(i \in[1, p])$. Then every graph $G$ with at least $d+p$ vertices and minimum degree at least d contains $F$ as a subgraph.

Our proof can be converted to a quadratic algorithm for embedding a forest. 
We consider simple undirected graphs without parallel edges and loops. The set of vertices adjacent to a vertex $x$, the neighborhood of $x$, is denoted $N(x)$. An embedding $f: H \rightarrow G$ of a graph $H$ in a graph $G$ is a one-to-one mapping $f: V(H) \rightarrow V(G)$ such that for any two distinct vertices $x, y \in V(H)$, if $x y \in E(H)$ then $f(x) f(y) \in E(G)$. For a graph $H$, the order of $H$ is the number of its vertices (denoted $|H|$ ) and the size of $H$ is is the number of its edges. For the terms not defined in this paper see ([10]).

\section{A Proof of Theorem 1}

We prove the theorem by induction on $p$, the number of trees in the forest. We can assume that every tree in a forest has at least two vertices, so $a_{i} \geq 1$.

The Base Case, $p=1$. The forest in this case consists of a single tree $T_{1}$ with $d$ edges. We prove a slightly stronger statement, which implies the theorem for $p=1$.

Lemma 1 Given a connected subgraph $C$ of $T_{1}$ and an embedding $f: C \rightarrow G$, there is an embedding $g: T_{1} \rightarrow G$ whose restriction to $C$ is precisely $f$.

Proof: The idea is to arbitrarily grow the embedding $f$ of $C$ to an embedding $g$ of $T_{1}$. If $|C|<d+1$, let $u v \in E\left(T_{1}\right)$ be an edge such that $u \in V(C)$ and $v \in V\left(T_{1} \backslash C\right)$. Let $w=f(u)$. Since $C$ has at most $d-1$ vertices other than $u$ and since the degree of $w$ in $G$ is at least $d, G$ has an edge $w z$ with vertex $z$ not in $g(C)$. Thus, $f$ can be expanded to $g: C \cup\{v\} \rightarrow G$ by defining $g(x)=g(x)$ for all $x \in C$, and $g(v)=z$. Iterating this expansion completes the proof.

Corollary 1 For any vertex $x$ of $T_{1}$ and any vertex $y$ of $G$, an embedding $f: T_{1} \rightarrow G$ exists for which $f(x)=y$.

The Induction Step, $p>1$. Assume the theorem holds for any forest $F_{p-1}$ with $p-1$ trees, and let $F_{p}=T_{1} \cup \cdots \cup T_{p}$ be a forest containing $p$ trees. Denote by $a_{i}$ the size of $T_{i}(i \in[1, p])$. Assume $a_{1} \geq a_{2} \geq \ldots \geq a_{p}$, and let $a=a_{1}$.

Assumption. For the purpose of deriving a contradiction, we assume that $F_{p}$ cannot be embedded in graph $G$ satisfying the conditions of the theorem.

Lemma 2 For every embedding $g: T_{1} \rightarrow G$, there is a vertex outside of $g\left(T_{1}\right)$ which is adjacent to every vertex in $g\left(T_{1}\right)$.

Proof: If the statement were incorrect, then the removal of $g\left(T_{1}\right)$ from $G$ would leave a subgraph $G^{\prime}$ with at least $d+p-(a+1)=\sum_{i=2}^{p}\left(1+a_{i}\right)$ vertices each of degree at least $d-a=\sum_{i=2}^{p} a_{i}$. Inductively, $T_{2} \cup \cdots \cup T_{p}$ can be embedded in $G^{\prime}$ which would yield an embedding of $F_{p}$ in $G$ contradicting the assumption that $F_{p}$ cannot be embedded in $G$. 
The main use of the previous lemma is to show that under our assumption, there is a large clique in $G$.

Lemma 3 G contains a clique of order at least a +2 .

Proof: Let $K$ be a largest clique in $G$ and suppose $|K|<a+2$. Select any connected subgraph $C$ of $T_{1}$ of order $|C|=|K|$, and embed $C$ in $K$; this is possible since $K$ is a clique. By Lemma 1 , this embedding can be expanded to an embedding $f$ of $T_{1}$ in $G$, and by Lemma 2 there is a vertex outside of $f\left(T_{1}\right)$ adjacent to all vertices in $f\left(T_{1}\right)$. In particular, it is adjacent to all vertices in $K$, contradicting $K$ 's maximality. Thus, $|K| \geq a+2$.

It turns out that for the rest of the proof, we only need a clique of order $a$.

Lemma 4 Any tree of order $a+1$ can be embedded in any connected graph of order at least $a+1$ that contains a clique of order $a$.

Proof: Start by embedding a leaf at a vertex outside an $a$-clique, but adjacent to a node in the clique (such a vertex must exist by connectivity). The remainder of the tree can be embedded in the clique.

Let $K$ be a clique of order $a$ in $G$. The subgraph $G^{\prime}=G \backslash K$ contains at least $d-a+p$ vertices each of degree at least $d-a$. Inductively, $F_{p-1}=\left\{T_{2}, \ldots, T_{p}\right\}$ can be embedded in $G^{\prime}$. Let $g: F_{p-1} \rightarrow G^{\prime}$ be such an embedding. Select any vertex $x \in K$ and a subset $X \subseteq N(x) \backslash K$ with $|X|=d-a+1$ vertices. It is possible since $|N(x) \backslash K| \geq d-a+1$.

Lemma 5 Every vertex in $X$ is used by any embedding $g$ of $F_{p-1}$.

Proof: Indeed, if $x \in X \backslash g\left(T_{p-1}\right)$ is not used, then by Lemma 4, $T_{1}$ can be embedded in the subgraph $H$ induced by $V(K) \cup\{x\}$, which would yield an embedding of $F_{p}$.

Since all $d-a+1$ vertices of $X$ are used in the embedding $g: F_{p-1} \rightarrow G$, exactly $p-2$ vertices outside of $K \cup X$, denoted by the set $Y(|Y|=p-2)$, are used by $g$. The remaining vertices of the graph, outside of $K \cup g\left(T_{p-1}\right)$, are denoted by the set $S ;|S|>0$ because $\left|K \cup g\left(T_{p-1}\right)\right|=d+p-1$ and $G$ has at least $d+p$ vertices. We now split the set of the trees of the forest $F_{p-1}$ into four subsets $\mathcal{T}_{1}, \mathcal{T}_{2}, \mathcal{T}_{3}$, and $\mathcal{T}_{4}$.

$\mathcal{T}_{1}$ : trees which are embedded entirely in $X$;

$\mathcal{T}_{2}$ : trees whose embedding has at least two vertices in $X$ and at least one vertex in $Y$;

$\mathcal{T}_{3}$ : trees whose embedding has only one vertex in $X$; and

$\mathcal{T}_{4}$ : trees whose embedding is entirely in $Y$.

Let $q_{i}=\left|\mathcal{T}_{i}\right|(i=1,2,3,4)$. Since every tree in $F_{p-1}$ belongs to exactly one of these four subsets,

$$
q_{1}+q_{2}+q_{3}+q_{4}=p-1 .
$$

For the embedding $g$ : every tree in $\mathcal{T}_{2}$ uses at least one vertex in $Y$; and, every tree $T_{i}$ in $\mathcal{T}_{3}$ $\left(\right.$ resp. $\left.\mathcal{T}_{4}\right)$ uses $a_{i}$ (resp. $1+a_{i}$ ) vertices in $Y$. Since there are $p-2$ vertices in $Y$,

$$
q_{2}+\sum_{T_{i} \in \mathcal{T}_{3}} a_{i}+\sum_{T_{i} \in \mathcal{T}_{4}}\left(a_{i}+1\right) \leq p-2=q_{1}+q_{2}+q_{3}+q_{4}-1 .
$$

This immediately gives a lower bound for $q_{1}$. 
Lemma $6 q_{1} \geq 1+\sum_{T_{i} \in \mathcal{T}_{3}}\left(a_{i}-1\right)+\sum_{T_{i} \in \mathcal{T}_{4}} a_{i} \geq 1+q_{4}$.

Let $s$ be an arbitrary vertex in $S$. Our goal now is to evaluate the degree of $s$ in the subgraph induced on $S$, based on the assumption that $F_{p}$ cannot be embedded. We start with

$$
|N(s) \cap S| \geq d-|N(s) \cap K|-|N(s) \cap(X \cup Y)| .
$$

We make the following observations about the neighborhood of $s$ in $K \cup X \cup Y$.

1. $s$ is not adjacent to any vertex in $K$, else by Lemma 4, $T_{1}$ could be embedded in $s \cup K$.

2. $s$ is not adjacent to at least one vertex in $g(T)$ for any tree $T \in \mathcal{T}_{2} \cup \mathcal{T}_{3}$. Indeed, if $s$ is adjacent to every vertex in $g(T)$, a vertex of $g(T)$ which is in $X$ can be swapped with $s$; this gives an embedding of $F_{p-1}$ that doesn't use every vertex of $X$, contradicting Lemma 5 .

3. $s$ is not adjacent to at least two vertices of $g(T)$ for any tree $T \in \mathcal{T}_{1}$. Indeed, suppose $s$ is adjacent to all but one vertex in $g(T)$, and let $y=g(x)$ be that exceptional vertex. Then for every neighbor $x^{\prime}$ (in $T$ ) of $x, s$ is adjacent to $g\left(x^{\prime}\right)$. By setting $g(x)=s$, we obtain a valid embedding of $F_{p-1}$ which doesn't use a vertex in $X$, contradicting Lemma 5.

So, $N(s) \cap K=\emptyset$ and $|N(s) \cap(X \cup Y)| \leq|X \cup Y|-\left(2 q_{1}+q_{2}+q_{3}\right)$. Since $|X \cup Y|=d-a+p-1$, we have from Inequality (1) that the number of neighbors of $s$ in $S$ is at least:

$$
\begin{aligned}
|N(s) \cap S| & \geq d-(d-a+p-1)+2 q_{1}+q_{2}+q_{3} \\
& =a+q_{1}-q_{4} \\
& \geq a+1
\end{aligned}
$$

where we have used $q_{1}+q_{2}+q_{3}+q_{4}=p-1$ and Lemma 6 . Thus, the degree of any vertex $s$ in the subgraph induced by $S$ is at least $a+1$, and in particular $|S| \geq a+2$. By Lemma $1, T_{1}$ can be embedded in this subgraph, contradicting the Assumption, and completing the proof of Theorem 1.

\section{Conjecture}

When the number of vertices equals the lower bound $p+d$ and the minimum degree is at least $d$, then the Hajnal-Szemerédi theorem on equitable coloring $([4,5])$, applied to the complement of the graph, guarantees the existence of $p$ cliques each of order at least $\lfloor d / p\rfloor$. Thus, an arbitrary $p$ graphs of order at most $\lfloor d / p\rfloor$ can be simultaneously embedded in the graph. When the number of vertices increases, however, cliques are no longer guaranteed. Our result shows that one can simultaneously embed trees, even as the number of vertices grows, as long as the sum of the tree sizes is at most $d$.

Alternatively, one can ask whether a bound on the minimum degree is excessive to guarantee that a forest can be embedded. Indeed, we propose a natural extension to the conjecture by Erdős and Sós: 
Let $F=T_{1} \cup \cdots \cup T_{p}$ be a forest, and $d=\sum_{i=1}^{p} a_{i}$, where $a_{i}$ is the number of edges in the tree $T_{i}(i \in[1, p])$. Then every graph $G$ with at least $d+p$ vertices and average degree $>d-1$ contains a subgraph isomorphic to $F$.

For a single star, the conjecture clearly holds; but, even the extension to a collection of stars is not clear.

\section{References}

[1] S. Brandt and E. Dobson. The Erdős-Sós conjecture for graphs of girth 5. Discrete Mathematics, 150:411-414, 1996. (Selected Papers in Honour of Paul Erdôs on the Occasion of his 80th Birthday (Keszthely, 1993)).

[2] N. Eaton and G. Tiner. On the Erdős-Sós conjecture and graphs with large minimum degree. Ars Combinatoria, 95:373-382, 2010.

[3] P. Erdôs. Some problems in graph theory. In M. Fiedler, editor, Theory of Graphs and its Applications, pages 29-36. Academic Press, New York, 1965.

[4] A. Hajnal and E. Szemerédi. Proof of a conjecture of P. Erdős. In P. Erdős, A. Rényi, and V. Sós, editors, Combinatorial Theory and its Applications, pages 601-623. North Holland, London, 1970.

[5] H. Kierstead and A. V. Kostochka. A short proof of the Hajnal-Szemerédi theorem on equitable colorings. Combinatorics, Probability \& Computing, 17:265-270, 2008.

[6] A. McLennan. The Erdős-Sós conjecture for trees of diameter four. Journal of Graph Theory, 49(4):291-301, 2005.

[7] J.-F. Saclé and M. Woźniak. The Erdős-Sós conjecture for graphs without $C_{4}$. Journal of Combinatorial Theory, Series B, 70:367-372, 1997.

[8] A. Sidorenko. Asymptotic solution for a new class of forbidden $r$-graphs. Combinatorica, 9:207-215, 1989.

[9] M. Wang, G. Li, and A. Liu. A result of Erdôs-Sós conjecture. Ars Combinatorica, 55:123-127, 2000.

[10] D. B. West. Introduction to Graph Theory. Prentice Hall, Upper Saddle River, NJ, 2003. 